\section{The latest in ultrasonic scaling}

Are you looking for the very latest in Cavitron ultrasonic scaling systems? Now there's no need to buy straight away. Instead, why not spread the cost and rent a brand new Cavitron Select SPS, Cavitron Touch or Cavitron Jet Plus unit from Dentsply Sirona from just $£ 65.00+$ VAT per month?

This economical rental solution enables you to use the latest dental technology for an affordable monthly fee [the unit can be purchased at the end of the rental agreement]. The agreement is spread over three years, with an extended warranty from two to three years, and includes one free on-site Cavitron Health Check and maintenance update per year, giving you complete peace of mind throughout the rental period. You can choose from the following rental options:

- Cavitron Select SPS Ultrasonic Scaler $£ 65.00$ + VAT per month

- Cavitron Touch Ultrasonic Scaling System $£ 75.00+$ VAT per month

- Cavitron Jet Plus Air Polishing and Scaler unit with new Tap-On Technology $£ 100.00+$ VAT per month.

To find out more about Cavitron Rental contact Dentsply Sirona on 08000723313 or read more at dentsply.com/en-uk/news.html

Earn DENTSPLY Rewards $£$ s on all your preventive solution purchases at dentsplyrewards.co.uk and access free CPD webinars and product demonstrations at dentsplyacademy.co.uk.

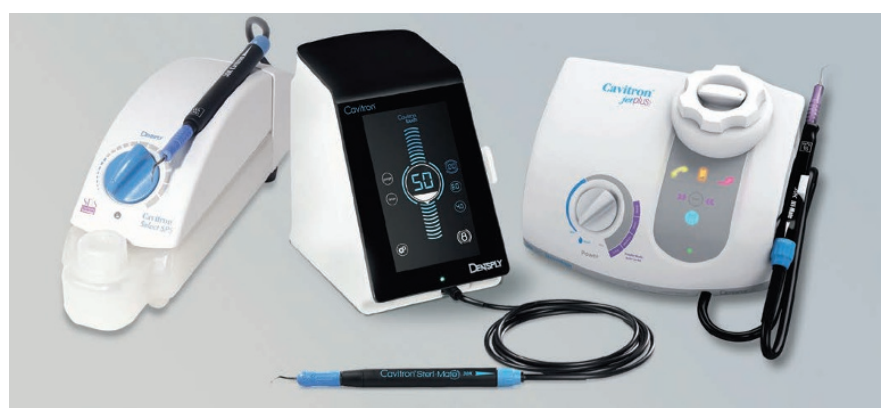

\section{Connect with your patients}

The team at Welltime knows just how important it is for you to develop a good dialogue with your patients - which is why their PatientConnections programme has been designed to allow for unprecedented levels of communications and feedback between any practice and its patients.

Easy to integrate into your existing website and simple to use, PatientConnections allows patients to leave feedback with ease - and makes that vital information available to you immediately. You will also be able to send your patients relevant articles and oral health tips to ensure they know as much about their own mouth as you do!

PatientConnections also has a dedicated Google Reviews function too, which helps put your practice on the map. You can manage your online reviews and use them to gain more visibility and enquiries.

When it comes to building a solid relationship with your patients, communication is key. Connect with them with ease through PatientConnections from Welltime. Contact the expert team today.

For more information, contact the Welltime team on 07999991 337, email sales@welltime.co.uk or visit www.welltime.co.uk.

\section{A soothing, moisturising rinse for dry mouth}

The Breath Company has launched a revolutionary dry mouth rinse that contains natural flower derivatives and moisturisers. The Breath Company Dry Mouth Rinse is clinically proven to be naturally effective at controlling and soothing dry mouth conditions without drying alcohol or staining compounds.

In clinical tests $95.4 \%$ of users said that the Dry Mouth Rinse helped reduce dryness and soothed their mouth, 97.1\% strongly agreed it was very refreshing, $96.2 \%$ said it moisturised their mouth and $75 \%$ of users said they would switch from their previous mouthwash.

Combining natural moisturisers, salivary enzymes and a salivary stimulant, The Breath Company Dry Mouth Rinse works to reverse the signs of xerostomia and help reduce the frequency of recurring dry mouth symptoms by stimulating, lubricating, moisturising, soothing and refreshing the mouth for hours.

The Breath Company Dry Mouth Rinse was scientifically developed in the US by Dr Harold Katz, the founder of the California Breath Clinics, and internationally acclaimed dentist/bacteriologist.

The Breath Company Dry Mouth Rinse is available in Boots stores nationwide and online at www.boots.com, priced at $£ 14.00$.

\section{A daily oral deodorant and enhanced hygiene}

Volatile sulphur compounds (VSCs) account for approximately $90 \%$ of the total sulphur content of mouth air and are the main culprits of halitosis. Not only that, recent research has suggested that VSCs may play an important role in periodontal aetiology and could be one of the contributing factors of carcinogenesis.

So, how can you help your patients tackle them? It's simple - recommend CB12 mouthwash.

CB12 has a unique patented formula containing zinc, that helps restore mucosa to a healthy state, combined with chlorhexidine diacetate, which can effectively convert offensive smelling VSCs to odourless, insoluble sulphides with long-lasting effects.

Available in two refreshing flavours, mint menthol and mild mint menthol, your patients can use CB12 as a daily oral deodorant to enhance oral hygiene levels and prevent oral malodour for up to 12 hours.

CB12 offers the vital combination of active ingredients, clinically proven to target and neutralise VSCs and help patients to achieve a fresh and healthy mouth.

For more information about CB12 and how it could benefit your patients, visit www.cb12.co.uk. 